\title{
Analysis of the Population Collision Process of Seeder Based on Linear Viscoelastic Model
}

\author{
Bin $\mathrm{Li}^{1, *}$, Jingjing Liu ${ }^{2}$ and Han-Jun $\mathrm{Ma}^{1}$ \\ ${ }^{I}$ School of Food Science, Henan Institute of Science and Technology, Xinxiang, 453003, China; \\ ${ }^{2}$ Xinxiang University, Xinxiang, 453003, China
}

\begin{abstract}
According to the linear viscoelastic model, the population discrete elements under the vibration working condition of the seed tray of the seeder were assumed as rigid conditions to analyze the collision process. The dynamic characteristics among the population collision process for the vibration process of the seeder were analyzed by tracing the microcosmic motion of each seed. The following conclusions were made, including the relationship between population velocity, phase angle as well as coefficient of restitution and the vibration intensity, the relationship between the center-ofgravity position as well as volume expansion coefficient of the population after collision and the vibration period, the relationship between the center-of-gravity position as well as volume expansion coefficient of the population after collision and the vibration frequency. This provides basis for determining the vibration work parameters of the precise vibration seeder. Based on agricultural machinery design experience and air-suction vibration principles, a new test bench for precise vibration air-suction tray seed metering device was researched by electromechanical integration technology. Performance test was carried out with oilseed rapeseeds as the research object. The test results show that when the vibration frequency is $10.5 \mathrm{~Hz}$, the vibration amplitude is $4 \mathrm{~mm}$, the vacuum negative pressure value is $3 \mathrm{kPa}$, the rotating velocity of the discharge plate is near $15 \mathrm{r} / \mathrm{min}$, and the pass index of the seeder will exceed $96 \%$, which provides basis for the structural adjustment and work parameter optimization of the seeder.
\end{abstract}

Keywords: Pass index, seeder, vacuum negative pressure value, vibration amplitude, vibration frequency.

\section{INTRODUCTION}

The seeds of agricultural crops are generally granular, which mechanical characteristics are between solid and fluid. Under natural stacking status, there is complicated squeezing and friction among the seeds, and then reasonably regulating the vibration parameters of the seed tray can make the seeds present "boiling" motion status, which can significantly reduce the seed suction resistance. To reduce miss-seeding and reseeding indexes and reduce the test strength in farmland, it's necessary to carry out dynamic analysis of the population collision process under working condition of the seeder.

Currently, abundant achievements have been made in terms of theory, numerical simulation and experiment research of the collision of single-degree-of-freedom system. Shaw [1] firstly researched the vibrators with unilateral constraint under simple harmonic excitation, analyzed the local intersection of periodic motion by Center Manifold Theorem, and analyzed chaotic motion by homoclinic phase intercept condition. Nordmark [2, 3] researched glancing collision phenomenon in details by building Poincare mapping of collision vibrators, and found that it was the main reason that caused the vibration system directly enter into chaotic motion from periodic motion. Salapaka et al. [4] carried out theoretical and experimental research on the motion of

*Address correspondence to this author at the School of Food Science, Henan Institute of Science and Technology, Xinxiang, 453003, China; E-mail: later@126.com spring vibrators under forced vibration, and observed the various motion forms of the system under inelastic collision, as well as verified the sensitivity of the stable periodic motion of the system to parameters. Akhavan [5] carried out experimental research on the complicated dynamic behaviors of Hertz's contact collision vibration system under different excitations.

Compared with single-degree-of-freedom system, the collision vibration problems of continuous system and multiple-degree-of-freedom system are more complicated. By far, there are only some theoretical achievements made in terms of research on collision and vibration of two-degreeof-freedom system $[6,7]$.

In this paper, the population discrete elements under the vibration working condition of the seed tray of the seeder were assumed as rigid conditions to analyze the collision process. The dynamic characteristics of the population collision process for the vibration process of the seeder were analyzed by tracing the microcosmic motion of each seed. The following conclusions were made, including the relationship between population velocity, phase angle as well as coefficient of restitution and the vibration intensity, the relationship between the center-of-gravity position as well as volume expansion coefficient of the population after collision and the vibration period, the relationship between the centerof-gravity position as well as volume expansion coefficient of the population after collision and the vibration frequency. This provides basis for determining the vibration work parameters of the precise vibration seeder. Based on agricultur- 
al machinery design experience and air-suction vibration principles, a new test bench for precise vibration air-suction tray seed metering device was researched by electromechanical integration technology. Performance test was carried out with oilseed rapeseeds as the research object.

\section{LINEAR VISCOELASTIC MODEL}

In linear viscoelastic model, the discrete elements are assumed as rigid conditions, i.e., the shape and volume of each element stay unchanged during the calculation process, and the contact force is generated from the deformation of spring damping and the slide friction [8,9], as shown in Fig. (1).

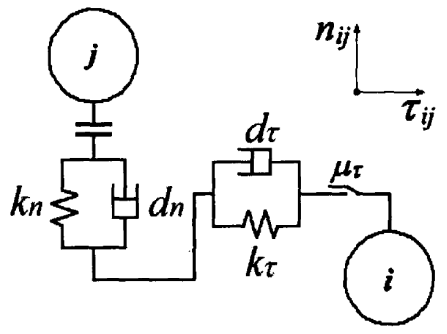

Fig. (1). Mechanical model of linear viscoelastic contact.

The normal and tangential components of the contact force can be indicated as [10]:

$f_{i j}^{n}=k_{n} \delta_{i j} n_{i j}-d_{n} v_{i j} n_{i j}$

$f_{i j}^{\tau}=-\min \left(k_{\tau} \xi_{i j}+d_{\tau} v_{i j}, \mu_{\tau}\left|f_{i j}^{n}\right|\right) \tau_{i j}$

In the equation (1), $k_{n}$ is normal contact rigidity, $k_{\tau}$ is tangential contact rigidity, $d_{n}$ is normal damping coefficient, $d_{\tau}$ is tangential damping coefficient, $\xi_{i j}$ is tangential displacement, $\mu_{\tau}$ is sliding friction coefficient among seeds, $\tau_{i j}$ is tangential unit vector and $v_{i j}$ is relative velocity.

\section{ANALYSIS OF THE POPULATION COLLISION PROCESS}

In this paper, the population motion simulation program was executed by means of explicit central difference method, i.e., with the displacement of the seeds as the basic variable, the resultant force and resultant moment applied on the seeds were obtained by the relationship between the force and displacement; the accelerated velocity and the angular acceleration were obtained through calculation according to Newton's Second Law of Motion; the kinematic parameters of each seed at any time were obtained through time integral; loop iteration was carried out by means of dynamic relaxation method; and the calculation was carried out according to time step length, with the position of each seed updated at each time step length. The law of the macroscopic motion of the entire population was acquired by tracing the microcosmic motion process of each seed. The flow is as shown in Fig. (2).

The simulation parameters were selected according to the general mechanical characteristics of oilseed rapeseeds: $\rho=1.4 \mathrm{~mm}, k_{n}=15000 \mathrm{~N} / \mathrm{m}, d_{n}=0.236 \mathrm{~N} \cdot \mathrm{m} / \mathrm{s}$, $k_{\tau}=10000 \mathrm{~N} / \mathrm{m}, \mu_{\tau}=0.2$,

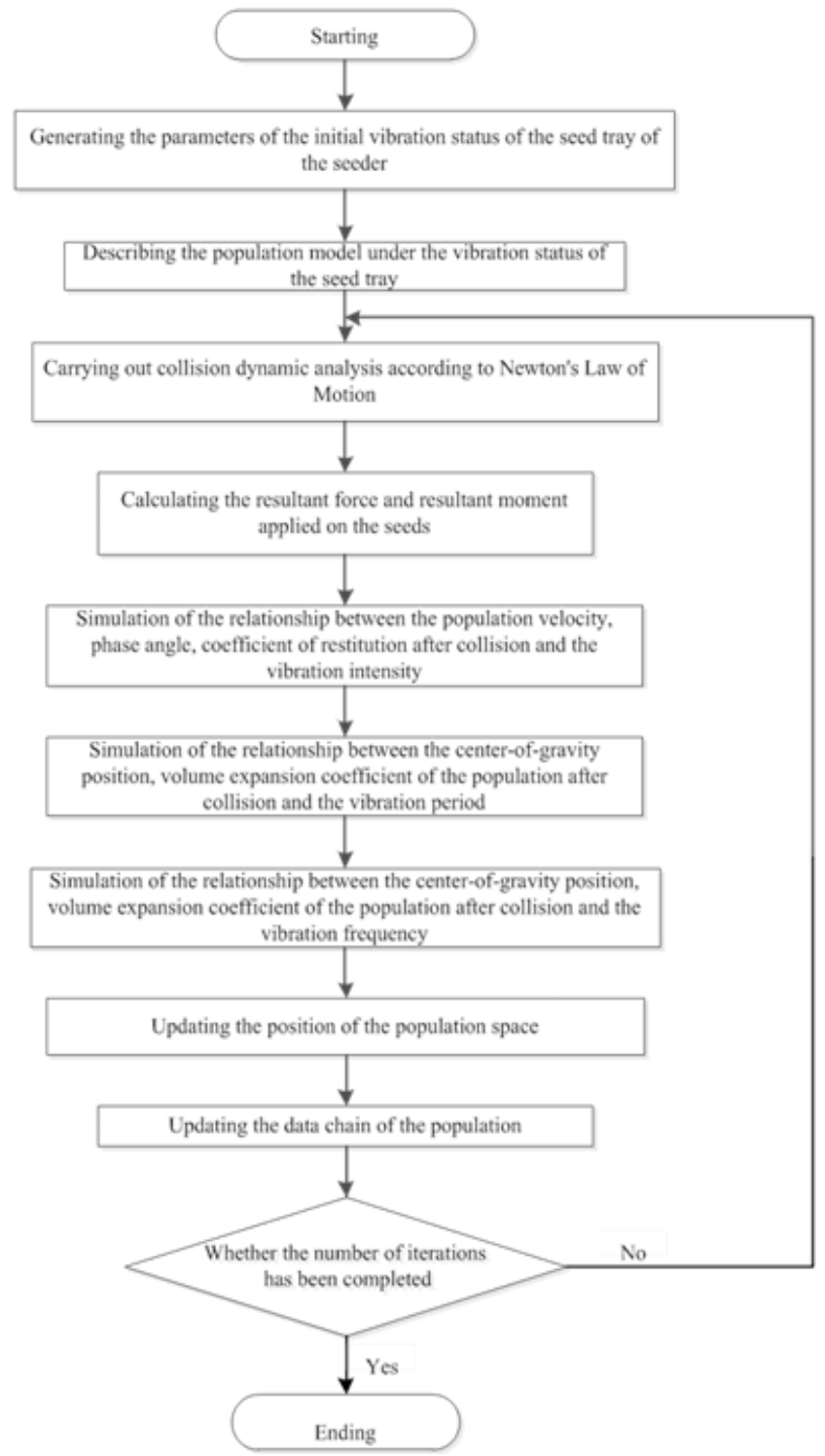

Fig. (2). Flow of analysis of the population collision.

$d_{\tau}=0.1927 \mathrm{~N} \cdot \mathrm{m} / \mathrm{s}, m=0.02 \mathrm{~g}$, the coefficient of restitution for the collision among seeds is $e=0.5$; the normal contact rigidity between the seeds and the seed tray is $30000 \mathrm{~N} / \mathrm{m}$; normal damping coefficient is $0.3338 \mathrm{~N} \cdot \mathrm{m} / \mathrm{s}$; tangential contact rigidity is $20000 \mathrm{~N} / \mathrm{m}$; tangential damping coefficient is $0.2725 \mathrm{~N} \cdot \mathrm{m} / \mathrm{s}$; coefficient of restitution of collision is $e=0.5$; friction coefficient is 0.3 ; and iteration time step length is $\Delta t=0.01 \mathrm{~ms}$.

The dynamic characteristics at the collision instant can be analyzed by simulating the collision between each two seeds, and the reasonability of the program and the parameter can be verified.

Fig. (3) shows the relationship between the velocity and the vibration intensity after collision between the seeds and the seed tray during the periodic collision. Fig. (4) shows the relationship between the collision phase angle and the vibration intensity. At the beginning of the periodic collision, the phase angle is $\delta=0$. With the increase of vibration intensity, 


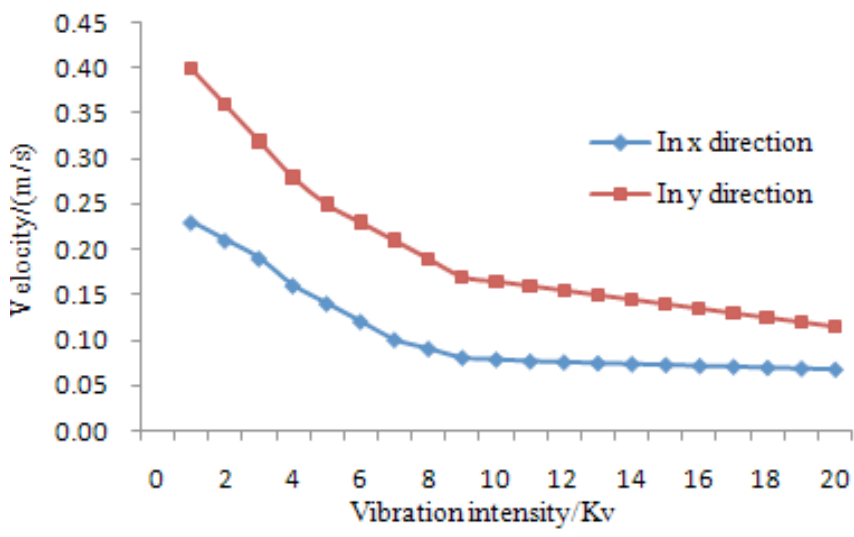

Fig. (3). The relationship between the velocity and the vibration intensity after collision.

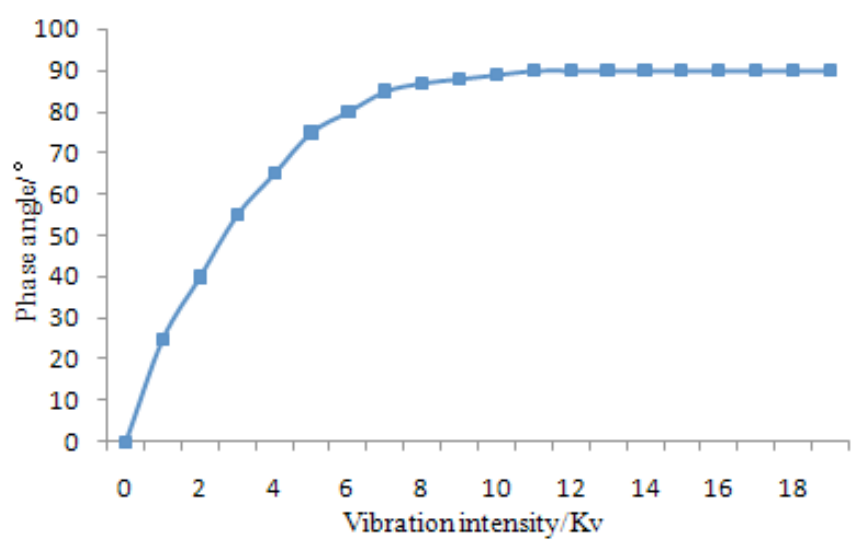

Fig. (4). The relationship between the collision phase angle and the vibration intensity.

$\delta$ gradually tends to $90^{\circ}$, and the velocity after collision between the seed tray and the seeds will be reduced.

Under the conditions of different coefficients of restitutions $e$ and mass ratios $\mu$, the vibration intensities corresponding to the periodic collisions are as shown in Figs. (5 and 6). When the vibration intensity $K_{v}>1$, the seeds can be tossed and collided with the table-board. However, the $K_{v}$ required by the periodic collision motion is closely related to $e$ and $\mu$. Moreover, $K_{v}$ increases with the reduction of $e$, and linearly increases with the increase of $\mu$.

Under natural stacking status, the population is often in discrete status due to internal friction, and it needs to be tamped by vibration, so as to make the seeds of the same quantity have the constant $h_{s}$. The motion status of the population is described by fluctuation coefficient of gravity center $G$ and volume expansion coefficient $H$, which are defined as:

$G(t)=\frac{1}{n} \sum_{i=1}^{n} z_{i}(\mathrm{t})$

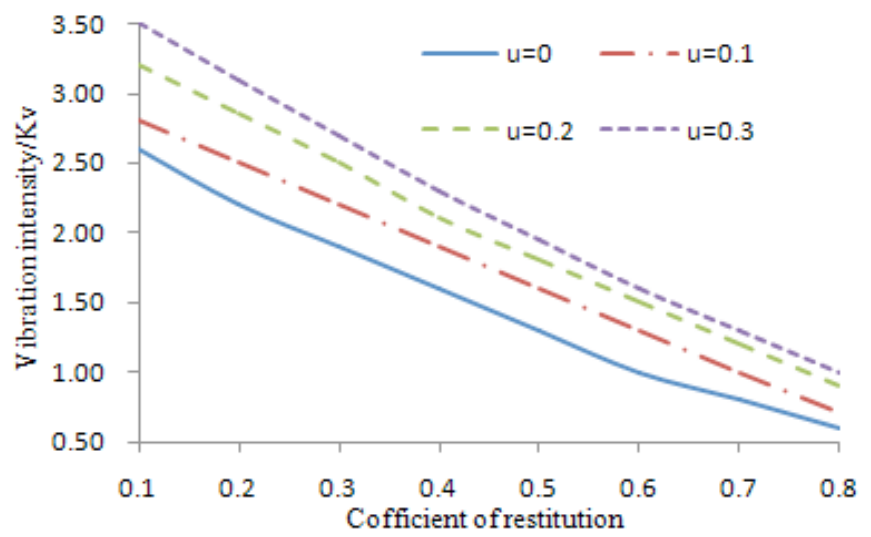

Fig. (5). Influence of coefficient of restitution on the periodic vibration.

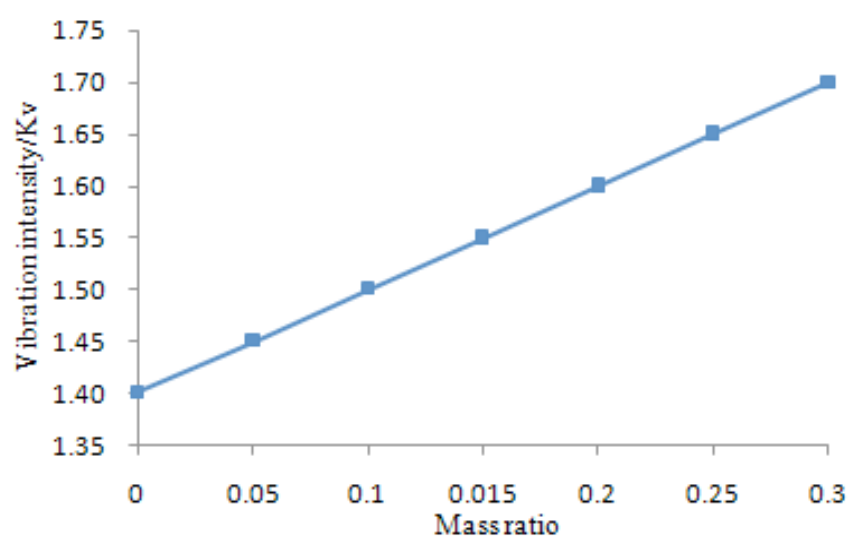

Fig. (6). The relationship between the mass ratio and the vibration intensity.

$H(t)=\frac{h_{v}(t)}{h_{s}}$

$h_{v}(t)=\frac{\sum_{i=1}^{n}\left(z_{i}-\min \left(z_{i}\right)+r\right)}{n r}$

The seed stray starts to excite from static status and the change processes of $G$ and $H$ are as shown in Figs. (7 and 8) respectively. It shows that $G$ and $H$ are both variables related to vibration parameters and time. Generally, the seeds can enter the approximate stable periodic status after 5 8 vibration periods.

When the vibration became stable, $G(t)$ and $H(t)$ signals were sampled by the frequency of $1 \mathrm{kHz}$ in the time of $2 \mathrm{~s}$. The average values of $G_{0}, H_{0}$ and harmonic components of fluctuation were acquired through FFT transform and analysis.

\section{PERFORMANCE ANALYSIS OF THE SEEDER}

With oilseed rapeseeds as the research object, performance test was carried out. Seeds with uniform thin layer were paved in the seed tray, and the initial thickness was 


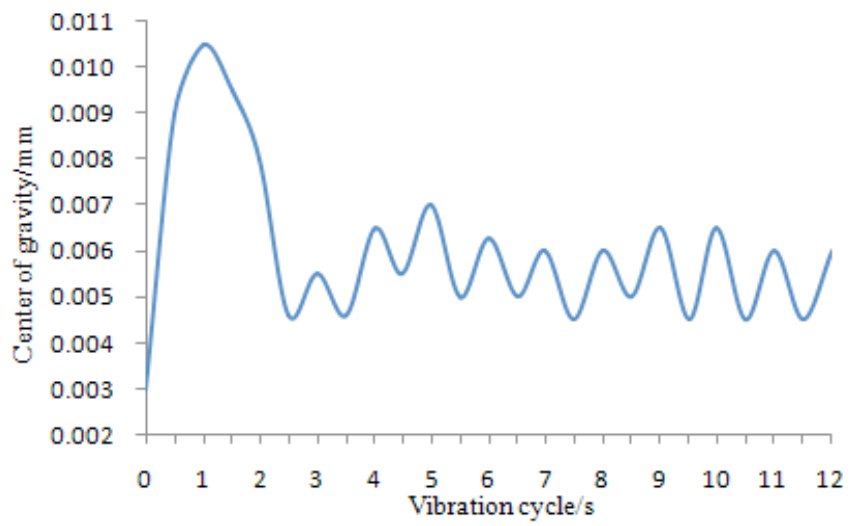

Fig. (7). Curve of change of population center of gravity.

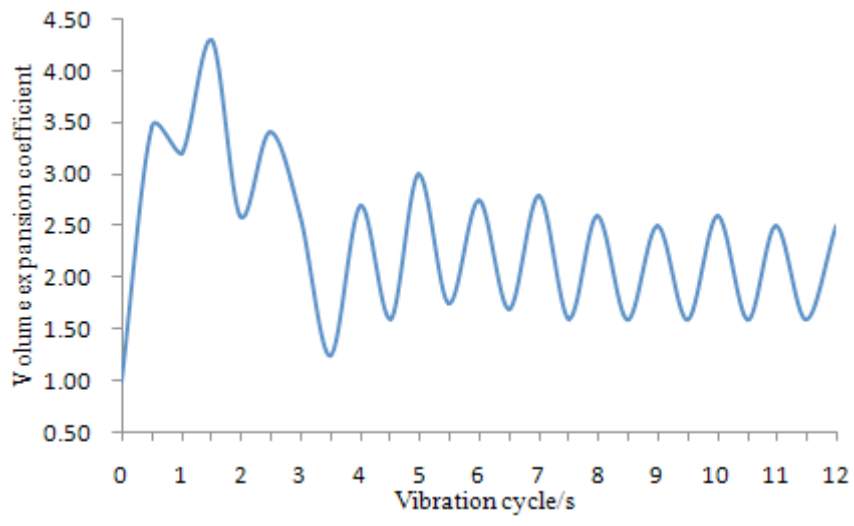

Fig. (8). Curve of change of volume expansion coefficient.

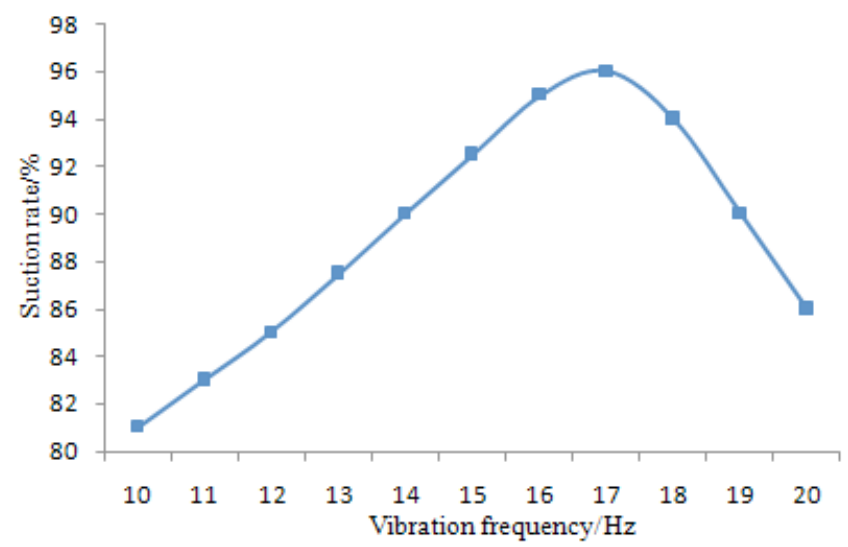

Fig. (9). The curve of the seed suction rate and the vibration frequency of the seed tray.

$h_{s} \approx 3.8$ as measured. The air pressure difference was regulated as $2 \mathrm{kPa}$ by a cone-shaped suction hole element with the diameter of $1 \mathrm{~mm}$. The vibration frequency range of the seed tray was $10 \sim 15 \mathrm{~Hz}$, the seed suction rate of the seeder is as shown in Fig. (9). It shows that the seed suction rate gradually increases with the increase of the vibration frequency of the seed tray. When the vibration frequency is $10.5 \mathrm{~Hz}$, the vibration amplitude is $4 \mathrm{~mm}$, the vacuum negative pressure value is $3 \mathrm{kPa}$, the rotating velocity of the discharge plate is near $15 \mathrm{r} / \mathrm{min}$, the seeds will be in "boiling" status, when the seed suction effect will be the best, with the seed suction rate reaching $96 \%$. With the further increase of the vibration frequency, the tossing height of the seeds will increase, and the density of the spatial distribution will reduce, so that the seed suction rate will show the obvious falling tend. The test results and theoretical analysis are basically consistent, thus verifying the correctness of the method.

\section{CONCLUSION}

According to the linear viscoelastic model, the population discrete elements under the vibration working condition of the seed tray of the seeder are assumed as rigid conditions to analyze the collision process among the seeds. The collision process of the population in the vibration seed tray of the precise vibration air-suction tray seeder is analyzed via Matlab software. The following conclusions are made, including the relationship between population velocity, phase angle as well as coefficient of restitution and the vibration intensity, the relationship between the center-of-gravity position as well as volume expansion coefficient of the population after collision and the vibration period, the relationship between the center-of-gravity position as well as volume expansion coefficient of the population after collision and the vibration frequency. This provides basis for determining the vibration work parameters of the precise vibration seeder. The seed tray is under small amplitude and high frequency vibration, which can make the seeds separated from each other, and the discrete degree increases with the increase of vibration intensity. The seed layer thickness has a significant influence on the motion law. The seeds in thinner layer are easier to separate. Performance test is carried out on the seeder with oilseed rapeseeds as the research object. The test results show that when the vibration frequency is $10.5 \mathrm{~Hz}$, the vibration amplitude is $4 \mathrm{~mm}$, the vacuum negative pressure value is $3 \mathrm{kPa}$, the rotating velocity of the discharge plate is near $15 \mathrm{r} / \mathrm{min}$, the pass index of the seeder will exceed $96 \%$, which provides basis for the structural adjustment and work parameter optimization of the seeder.

\section{CONFLICT OF INTEREST}

The authors confirm that this article content has no conflict of interest.

\section{ACKNOWLEDGEMENTS}

Supported by Program for Innovative Research Team (in Science and Technology) in University of Henan Province (Grant No. 13IRTSTHN006).

\section{REFERENCES}

[1] S. W. Shaw and P. J. Holmes, "A periodically forced piecewise linear oscillator," J. Vib., vol. 90, no. 1, pp. 129-155, 1983.

[2] A. B. Nordmark, "Universal limit mapping in grazing bifurcations," Physical Review E, vol. 55, no. 1, pp. 266-270, 1997.

[3] J. Bengtsson, W. K. Persson, and A. Kjellberg, "Evaluations of effects due to low-frequency noise in a low demanding work situation," J. Soun. Vib., vol. 278, no. 1, pp. 83-99, 2004.

[4] S. Salapaka, M. V. Salapaka, M. Dahleh, I. Mezic, "Complex dynamics in repeated impact oscillators decision and control," In: Proceedings of the 37th IEEE Conference on. IEEE, vol. 2, pp. 2053-2058, 1998.

[5] T. Akhavan, B. L Luhovyy, P. H. Brown, C.E. Cho, and G.H. Anderson, "Effect of premeal consumption of whey protein and its 
hydrolysate on food intake and post-meal glycemia and insulin responses in young adults," Am. J. Clin. Nutr., vol. 91, no. 4, pp. 966-975, 2010.

[6] T. Akhavan, B. L. Luhovyy, S. Panahi, R. Kubart, P.H. Brown, and G.H. Anderson, "Mechanism of action of pre-meal consumption of whey protein on glycemic control in young adults," J. Nutr. Biochem., vol. 25 , no. 1, pp. 36-43, 2014.

[7] D. Jakubowicz, and O. Froy, "Biochemical and metabolic mechanisms by which dietary whey protein may combat obesity and Type 2 diabetes," J. Nutr. Biochem., vol. 24, no. 1, pp. 1-5, 2013.

[8] Y. Q. Liu, M. Q. Zhao, and Y. W. Hu, "Finite element analysis of airflow field for vacuum chamber in air-suction seed metering de- vice of no-till seeder," Appl. Mech. Mater., vol. 275 pp. 547-553, 2013.

[9] D. Y. Geng, R. C. Du, D. L. Zhang, and L. Dan, "Experimental research on belt type of precision seed-metering device," $A d v$. Mater. Res., no. 712, pp. 1339-1342, 2013.

[10] R. C. Singh, G. Singh, and D. C. Saraswat, "Optimization of design and operational parameters of a pneumatic seed metering device for planting cottonseeds," Biosystems Eng., vol. 92, no. 4, pp. 429-438, 2005 .

(C) Li et al.; Licensee Bentham Open.

This is an open access article licensed under the terms of the Creative Commons Attribution Non-Commercial License (http://creativecommons.org/licenses/by-nc/4.0/) which permits unrestricted, non-commercial use, distribution and reproduction in any medium, provided the work is properly cited. 